\title{
Invariants for Smooth Conjugacy of Hyperbolic Dynamical Systems. I
}

\author{
J. M. Marco and R. Moriyón * \\ Department of Mathematics, Universidad Autónoma de Madrid, E-28049 Madrid, Spain
}

\begin{abstract}
We prove that the Lyapunov exponents of periodic orbits are a total family of invariants for $C^{\infty}$ conjugation of families of diffeomorphisms to a two-dimensional toral automorphism. In case of families of canonical diffeomorphisms, the corresponding conjugating diffeomorphisms are also canonical.
\end{abstract}

During the last few years there has been a considerable advance in perturbation theory of Anosov hamiltonian systems (see [GK, CEG, LMM]). In this paper we start the study of the corresponding $C^{\infty}$ conjugation problem for general (not necessarily hamiltonian) systems. We prove in some particular situations that the simplest conjugation invariants, the Lyapunov exponents of periodic orbits, are a total family of invariants for $C^{\infty}$ conjugation.

We also show that Lyapunov exponents are relevant to perturbation theory of hamiltonian Anosov systems. We prove that for hamiltonian systems of the class under consideration, the constancy of the Lyapunov exponents implies the constancy of the action invariants, which are known to be a complete family of invariants for canonical conjugation. This in turn implies that the Lyapunov exponents are (in our situation) a complete family of invariants for locally hamiltonian, as well as for hamiltonian, conjugation. We find this interesting since it is shown in [CEG] [see the comments following (1.9) and the example in Appendix E] that Lyapunov exponents are not a complete family of invariants for conjugation of locally hamiltonian perturbations of geodesic flows with negative curvature in space dimension 2 under globally canonical maps, and no positive result in this direction was known up to now. It is tempting to conjecture that our results can be extended to cover perturbations of suitable flows, at least in dimension 3.

We work in the context of one-parameter families of diffeomorphisms of the two-dimensional torus $T^{2}=\mathbb{R}^{2} / \mathbb{Z}^{2}$. Such a one-parameter family $f_{\varepsilon}, a<\varepsilon<b$, will

* Supported by CAICYT grant 397/84 
be called an isotopy if the map $(\varepsilon, x) \mapsto f_{\varepsilon}(x)$ is $C^{\infty}$. The generator of the isotopy is the (parameter depending) vector field $X_{\varepsilon}$ determined by

$$
\frac{d f_{\varepsilon}}{d \varepsilon}=X_{\varepsilon} \circ f_{\varepsilon}
$$

If each generator is locally hamiltonian (respectively hamiltonian) with respect to the usual symplectic form $\omega=d x \wedge d y$ we shall say that $f_{\varepsilon}$ is a locally hamiltonian isotopy (LHI) [respectively a globally hamiltonian isotopy (GHI)]. The hamiltonian $F_{\varepsilon}$ of $X_{\varepsilon}$ with vanishing average over $T^{2}$ is called the hamiltonian of the isotopy. Obviously, LHI's are just isotopies made up of canonical maps (area preserving maps in our case).

We shall use the basic theory of Anosov diffeomorphisms, as developed for example in [A] (where they are called $C$-cascades) or in [S], without any special mention of it.

Before stating our main result, we define the invariants. Assume $f: M \rightarrow M$ is a diffeomorphism of $M, x \in M$, and $f^{N}(x)=x$. The Lyapunov exponents of $f$ at the orbit of $x$ are the logarithms of the positive $N^{\text {th }}$ roots of the absolute values of the eigenvalues of the linear map $T_{x} f^{N}$. They measure the rate at which points near $x$ separate from the orbit of $x$ under iteration. Obviously, if $g=\varphi \circ f \circ \varphi^{-1}$ with a smooth $\varphi$, the Lyapunov exponents of $g$ at the orbit of $\varphi(x)$ are the same as those of $f$ at the orbit of $x$, so the Lyapunov exponents of periodic orbits are smooth conjugation invariants. Let us point out that Lyapunov exponents can be defined for almost any $x$ with respect to an invariant measure (see [P]), and they are still invariant under conjugation. For transitive Anosov systems, which have a dense set of periodic orbits, one expects the invariants of these orbits to contain all the relevant information for smooth conjugation. The theorem below is a specific fact in this direction.

If $f_{\varepsilon}$ is an isotopy and the periodic point $x_{0}$ of $f_{0}$ is hyperbolic, we can follow $x_{0}$ along the isotopy for small values of the parameter. If $f_{0}$ is uniformly hyperbolic (i.e. an Anosov system) all the periodic orbits can be followed along the same interval $-\varepsilon_{0}<\varepsilon<\varepsilon_{0}$, since such systems are persistent under perturbations. Thus, Lyapunov exponents are defined for $-\varepsilon_{0}<\varepsilon<\varepsilon_{0}$ and $x_{\varepsilon}$ corresponding to a periodic point $x_{0}$ of $f_{0}$.

Theorem. Assume $f_{\varepsilon},-1<\varepsilon<1$, is an isotopy (respectively an $L H I$ ) (respectively a GHI) on $T^{2}$, and $f_{0}$ is defined by a $2 \times 2$ hyperbolic integer matrix. Then there exists another isotopy (respectively an LHI), (respectively a GHI) $\varphi_{\varepsilon}$ satisfying $f_{0}=\varphi_{\varepsilon} \circ f_{\varepsilon} \circ \varphi_{\varepsilon}^{-1},-\varepsilon_{0}<\varepsilon<\varepsilon_{0}$, if and only if for any periodic point $x_{0}$ of $f_{0}$, the Lyapunov exponents of the corresponding $x_{\varepsilon}$ have a value independent of $\varepsilon$. Moreover, $\varphi_{0}=I$.

Remark 1. If we assume that each $f_{\varepsilon}$ is Anosov, then we can make $\varepsilon_{0}=1$.

Remark 2. This theorem has the flavour of Arnold's theorem on the conjugacy of diffeomorphisms of the circle to well behaved rotations (see [A, 12.A]). Its extension to more general systems, like arbitrary Anosov diffeomorphisms or flows and higher dimensions is an interesting problem. Soon after a first version of this paper was available, $R$. de la Llave has been able to substitute the isotopies $f_{\varepsilon}$ 
in our result by two single diffeomorphisms, $f_{0}$ and $f_{1}$, close enough, without any linearity assumption on them. His result appears in a subsequent paper, Invariants for Smooth Conjugacy of Hyperbolic Systems, II. Further extensions to flows and higher dimensions, some of them without closeness assumptions, will be the subject of successive papers in this series.

Remark 3. There are very strong conjugacy results for geodesic flows on manifolds of negative curvature, mainly related to the length spectrum instead of the Lyapunov exponents. See [BK, Sects. 3 and 10], for a recent survey on the subject.

The nontrivial part of the proof of the theorem is showing that the independence of the Lyapunov exponents on $\varepsilon$ implies that all the $f_{\varepsilon}$ 's are conjugate to $f_{0}$. This is proved with the help of the following three Lemmas:

Lemma 1. If $f_{\varepsilon},-\varepsilon_{0}<\varepsilon<\varepsilon_{0}$, is an isotopy of orientation preserving Anosov diffeomorphisms on $T^{2}, f_{0}$ is area preserving, and the Lyapunov exponents of corresponding periodic points $x_{\varepsilon}$ are independent of $\varepsilon$, there exists an isotopy $\psi_{\varepsilon}$, $-\varepsilon_{0}<\varepsilon<\varepsilon_{0}$, with $\psi_{0}=I$, such that $\psi_{\varepsilon} \circ f_{\varepsilon} \circ \psi_{\varepsilon}^{-1}$ is an LHI.

Lemma 2. If $g_{\varepsilon},-\varepsilon_{0}<\varepsilon<\varepsilon_{0}$, is an LHI on $T^{2}$, and $g_{0}$ is defined by a $2 \times 2$ hyperbolic integer matrix, then there exists an LHI $\eta_{\varepsilon},-\varepsilon_{0}<\varepsilon<\varepsilon_{0}$, with $\eta_{0}=I$, such that $\eta_{\varepsilon} \circ g_{\varepsilon} \circ \eta_{\varepsilon}^{-1}$ is a $G H I$.

Lemma 3. If $h_{\varepsilon},-\varepsilon_{0}<\varepsilon<\varepsilon_{0}$, is a GHI of Anosov diffeomorphisms on $T^{2}$ with hamiltonian $H_{\varepsilon}, h_{0}$ is defined by a $2 \times 2$ hyperbolic integer matrix, and the Lyapunov exponents of corresponding periodic points $x_{\varepsilon}$ do not depend on $\varepsilon$, then for any periodic point $x_{\varepsilon}$ of period $N$

$$
\sum_{i=0}^{N-1} H_{\varepsilon}\left(h_{\varepsilon}^{i}\left(x_{\varepsilon}\right)\right)=0 \text {. }
$$

The theorem is a consequence of Lemmas 1-3, and Theorem 1.3 in [LMM], which states that if (1) is satisfied, all the $h_{\varepsilon}$ 's are conjugated to $h_{0}$ by a GHI. We give now the proofs of the lemmas.

Proof of Lemma 1. As a consequence of the independency of the Lyapunov exponents with respect to $\varepsilon$, the determinant of $T_{x_{\varepsilon}} f_{\varepsilon}^{N}$ is independent of $\varepsilon$. ( $N$ denotes the period of $x_{\varepsilon}$.) Since $f_{0}$ preserves area, and orientation, the determinant is 1 . If we call $D_{\varepsilon}(x)=\log \operatorname{det} T_{x} f_{\varepsilon}$, we see that as a consequence of the chain rule, given $x_{\varepsilon}, N$ as above,

$$
\sum_{i=0}^{N-1} D_{\varepsilon}\left(f_{\varepsilon}\left(x_{\varepsilon}\right)\right)=0
$$

Each $f_{\varepsilon}$ is a transitive diffeomorphism, since it is conjugated to the area preserving map $f_{0}$. Thus, according to Theorems 2.1 and 2.2 in [LMM], there exists a $C^{\infty}$ family of $C^{\infty}$ functions $\xi_{\varepsilon},-\varepsilon_{0}<\varepsilon<\varepsilon_{0}$, such that $\xi_{0}=0$ and $D_{\varepsilon}=\xi_{\varepsilon}-\xi_{\varepsilon} \circ f_{\varepsilon}$. This implies that

$$
f_{\varepsilon *}\left(e^{\xi_{\varepsilon}} d x \wedge d y\right)=e^{\xi_{\varepsilon}} d x \wedge d y .
$$

If we consider $\eta_{\varepsilon}=\xi_{\varepsilon}+K_{\varepsilon}$ for a one-parameter family of constants $K_{\varepsilon}$, $-\varepsilon_{0}<\varepsilon<\varepsilon_{0}$, that will be fixed in a moment, we also have

$$
f_{\varepsilon *}\left(e^{\eta_{\varepsilon}} d x \wedge d y\right)=e^{\eta_{\varepsilon}} d x \wedge d y .
$$


Moreover,

$$
\begin{aligned}
\frac{d}{d \varepsilon} \int_{T^{2}} e^{\eta_{\varepsilon}} d x \wedge d y & =\int_{T^{2}} \frac{d \eta_{\varepsilon}}{d \varepsilon} \cdot e^{\eta_{\varepsilon}} d x \wedge d y \\
& =e^{K_{\varepsilon}}\left(\frac{d}{d \varepsilon} \int_{T^{2}} e^{\xi_{\varepsilon}} d x \wedge d y+\frac{d K_{\varepsilon}}{d \varepsilon} \int_{T^{2}} e^{\xi_{\varepsilon}} d x \wedge d y\right)
\end{aligned}
$$

So if we fix $K_{\varepsilon}$ with $K_{0}=0$, and

$$
\frac{d K_{\varepsilon}}{d \varepsilon}=-\left(\frac{d}{d \varepsilon} \int_{T^{2}} e^{\xi_{\varepsilon}} d x \wedge d y\right) /\left(\int_{T^{2}} e^{\xi_{\varepsilon}} d x \wedge d y\right),
$$

then the measure $d \mu_{\varepsilon}=e^{\eta_{\varepsilon}} d x \wedge d y$ is invariant under $f_{\varepsilon}$ and has total volume independent of $\varepsilon$.

By a well known theorem of Moser, [Mo], for each $\varepsilon$ there exists a $C^{\infty}$ diffeomorphism $\psi_{\varepsilon}$ such that $\psi_{\varepsilon *} d \mu_{\varepsilon}=d x \wedge d y$, and $\psi_{0}=I$. Since $\psi_{\varepsilon}$ depends on $\varepsilon$ in a $C^{\infty}$ way, so it is an isotopy. Then,

$$
\left(\psi_{\varepsilon} \circ f_{\varepsilon} \circ \psi_{\varepsilon}^{-1}\right)_{*}(d x \wedge d y)=\psi_{\varepsilon *} f_{\varepsilon *} d \mu_{\varepsilon}=\psi_{\varepsilon *} d \mu_{\varepsilon}=d x \wedge d y,
$$

and this shows that $\psi_{\varepsilon} \circ f_{\varepsilon} \circ \psi_{\varepsilon}^{-1}$ is an LHI. The proof of the lemma is finished.

Remark. The above proof can immediately be extended to the higher dimensional case: if $f_{\varepsilon}$ is an isotopy of Anosov diffeomorphisms on an $n$-dimensional manifold $M, \omega_{0} \in \Lambda^{n} M$ is a volume form, invariant under $f_{0}$, and the Lyapunov exponents of corresponding periodic points $x_{\varepsilon}$ of $f_{\varepsilon}$ are independent of $\varepsilon$, then there exists an isotopy $\psi_{\varepsilon}$ with $\psi_{0}=I$, such that $\omega_{0}$ is invariant under $\psi_{\varepsilon} \circ f_{\varepsilon} \circ \psi_{\varepsilon}^{-1}$ for any $\varepsilon$.

Proof of Lemma 2. It is a direct consequence of Theorem 1.2 in [LMM], which says that whenever $\mathrm{Id}-g_{0 \#}$ is an isomorphism on $H^{1}\left(T^{2}\right)$, and $g_{\varepsilon}$ is an LHI of Anosov diffeomorphisms, they can be conjugated by an LHI to a GHI.

Remark. Lemma 2 is true in any dimension without the assumption on $g_{0}$ since by $[\mathrm{M}]$, each $g_{\varepsilon}$ is topologically conjugate to a diffeomorphism defined by a hyperbolic matrix.

The proof of Lemma 3 is based on the following lemma:

Lemma 4. There exist one-parameter families of closed one-forms $\alpha_{\varepsilon}^{s}, \alpha_{\varepsilon}^{u}$, $-\varepsilon_{0}<\varepsilon<\varepsilon_{0}$, of class $C^{1+\delta}$ as functions of $(\varepsilon, x) \in\left(-\varepsilon_{0}, \varepsilon_{0}\right) \times T^{2}$, such that

$$
\begin{aligned}
& h_{\varepsilon}^{*} \alpha_{\varepsilon}^{s}=\lambda^{s} \alpha_{\varepsilon}^{s}, \\
& h_{\varepsilon}^{*} \alpha_{\varepsilon}^{u}=\lambda^{u} \alpha_{\varepsilon}^{u},
\end{aligned}
$$

where the constants $\lambda^{s}, \lambda^{u}$ are the eigenvalues of the matrix that defines $h_{0}, 0<\left|\lambda^{s}\right|$ $<1<\left|\lambda^{u}\right|$. Moreover,

$$
\alpha_{\varepsilon}^{s} \wedge \alpha_{\varepsilon}^{u}=d x \wedge d y,
$$

and $\left[\alpha_{\varepsilon}^{s}\right],\left[\alpha_{\varepsilon}^{u}\right] \in H^{1}\left(T^{2}, \mathbb{R}\right)$ are independent of $\varepsilon$.

Lemma 4 is very natural, since in the case $\varepsilon=0$ it is a trivial consequence of our assumption regarding $h_{0}$. It actually gives a lot of information about the dynamics of each $h_{\varepsilon}$, except for the regularity of the stable and unstable foliations. 
We shall assume Lemma 4 for the moment, and we will see now how Lemma 3 can be deduced from it. The main idea is that differentiating (a) with respect to $\varepsilon$ gives an expression for $H_{\varepsilon}$ from which (1) can be proved explicitly. One of the main points of the proof is that we never perform more derivatives on $\alpha_{\varepsilon}^{s}, \alpha_{\varepsilon}^{u}$ than is allowed, namely either one derivative along the parameter or along the space variable.

Proof of Lemma 3. Let $V_{\varepsilon}^{s}, V_{\varepsilon}^{u}$ be the dual basis of $\alpha_{\varepsilon}^{s}, \alpha_{\varepsilon}^{u}$. For any $C^{1}$ function $G$ on $T^{2}$ we have

$$
d G=\left(V_{\varepsilon}^{s} G\right) \alpha_{\varepsilon}^{s}+\left(V_{\varepsilon}^{u} G\right) \alpha_{\varepsilon}^{u} .
$$

By (b), if $h_{\varepsilon}$ is generated by the hamiltonian vector field $X_{\varepsilon}$ with hamiltonian $H_{\varepsilon}$,

$$
d H_{\varepsilon}=\alpha_{\varepsilon}^{s}\left(X_{\varepsilon}\right) \alpha_{\varepsilon}^{u}-\alpha_{\varepsilon}^{u}\left(X_{\varepsilon}\right) \alpha_{\varepsilon}^{s},
$$

so by (3) we get

$$
V_{\varepsilon}^{u} H_{\varepsilon}=\alpha_{\varepsilon}^{s}\left(X_{\varepsilon}\right), \quad V_{\varepsilon}^{s} H_{\varepsilon}=-\alpha_{\varepsilon}^{u}\left(X_{\varepsilon}\right) .
$$

Differentiating in (a) with respect to $\varepsilon$ we have

$$
h_{\varepsilon}^{*}\left(d i_{X_{\varepsilon}} \alpha_{\varepsilon}^{s}+\frac{d \alpha_{\varepsilon}^{s}}{d \varepsilon}\right)=\lambda^{s} \cdot \frac{d \alpha_{\varepsilon}^{s}}{d \varepsilon},
$$

and similarly for $\alpha_{\varepsilon}^{u}$. If we evaluate the previous expression on $V_{\varepsilon}^{s}$ we are led to

$$
\left[V_{\varepsilon}^{s}\left[\alpha_{\varepsilon}^{s}\left(X_{\varepsilon}\right)\right]\right] \circ h_{\varepsilon}+\frac{d \alpha_{\varepsilon}^{s}}{d \varepsilon}\left(V_{\varepsilon}^{s} \circ h_{\varepsilon}\right)=\frac{d \alpha_{\varepsilon}^{s}}{d \varepsilon}\left(V_{\varepsilon}^{s}\right) .
$$

This gives by (4)

$$
V_{\varepsilon}^{s} V_{\varepsilon}^{u} H_{\varepsilon}=\frac{d \alpha_{\varepsilon}^{s}}{d \varepsilon}\left(V_{\varepsilon}^{s} \circ h_{\varepsilon}^{-1}\right)-\frac{d \alpha_{\varepsilon}^{s}}{d \varepsilon}\left(V_{\varepsilon}^{s}\right) .
$$

This formula looks close to what we need, since the right-hand side is of the form $\eta_{\varepsilon} \circ h_{\varepsilon}^{-1}-\eta_{\varepsilon}$. If we knew how to solve the equations $V_{\varepsilon}^{s} \bar{\eta}_{\varepsilon}=\eta_{\varepsilon}$ and $V_{\varepsilon}^{u} \tilde{\eta}_{\varepsilon}=\bar{\eta}_{\varepsilon}$ we would have $V_{\varepsilon}^{s} V_{\varepsilon}^{u}\left(H_{\varepsilon}-\tilde{\eta}_{\varepsilon} \circ h_{\varepsilon}^{-1}+\tilde{\eta}_{\varepsilon}\right)=0$, and then $H_{\varepsilon}=\tilde{\eta}_{\varepsilon} \circ f_{\varepsilon}-\tilde{\eta}_{\varepsilon}$, and (1) would follow immediately. In the particular case $\varepsilon=0$ both equations can be solved explicitly (up to constants) by Fourier Analysis, since they are first order PDE's with constant coefficients, but for arbitrary $\varepsilon$, Lemma 4 does not give us enough regularity on $\alpha_{\varepsilon}^{s}, \alpha_{\varepsilon}^{u}$ (hence on $V_{\varepsilon}^{s}, V_{\varepsilon}^{u}$ ) as to solve them. However, we can still use the remaining information in Lemma 4 , in order to prove that $H_{\varepsilon}$ has the desired form as follows.

Since $\left[\alpha_{\varepsilon}^{s}\right],\left[\alpha_{\varepsilon}^{u}\right] \in H^{1}(M, \mathbb{R})$ are independent of $\varepsilon$, there exist functions $G_{\varepsilon}^{s}, G_{\varepsilon}^{u}$ of class $C^{1+\delta}$ on $T^{2}$ for each fixed $\varepsilon$ such that

$$
\frac{d \alpha_{\varepsilon}^{s}}{d \varepsilon}=d G_{\varepsilon}^{s},
$$

and similarly for $\alpha_{\varepsilon}^{u}$ and $G_{\varepsilon}^{u}$, and we shall have

$$
d\left(G_{\varepsilon}^{s} \alpha_{\varepsilon}^{u}-G_{\varepsilon}^{u} \alpha_{\varepsilon}^{s}\right)=\frac{d \alpha_{\varepsilon}^{s}}{d \varepsilon} \wedge \alpha_{\varepsilon}^{u}-\frac{d \alpha_{\varepsilon}^{u}}{d \varepsilon} \wedge \alpha_{\varepsilon}^{s}=\frac{d}{d \varepsilon}\left(\alpha_{\varepsilon}^{s} \wedge \alpha_{\varepsilon}^{u}\right)=\frac{d}{d \varepsilon}(d x \wedge d y)=0 .
$$


Since $\left[\alpha_{\varepsilon}^{s}\right],\left[\alpha_{\varepsilon}^{u}\right]$ are a basis of $H^{1}(M, \mathbb{R})$, by adding suitable constants to $G_{\varepsilon}^{s}, G_{\varepsilon}^{u}$ we can achieve that $G_{\varepsilon}^{s} \alpha_{\varepsilon}^{u}-G_{\varepsilon}^{u} \alpha_{\varepsilon}^{s}$ is exact. Then there exists a function $G_{\varepsilon}$ of class $C^{2+\delta}$ for fixed $\varepsilon$, such that

By (3) this gives

$$
G_{\varepsilon}^{s} \alpha_{\varepsilon}^{u}-G_{\varepsilon}^{u} \alpha_{\varepsilon}^{s}=d G_{\varepsilon}
$$

$$
V_{\varepsilon}^{s} G_{\varepsilon}=-G_{\varepsilon}^{u}, \quad V_{\varepsilon}^{u} G_{\varepsilon}=G_{\varepsilon}^{s} .
$$

Substituting (6) and (7) into (5) we obtain

$$
V_{\varepsilon}^{s} V_{\varepsilon}^{u} H_{\varepsilon}=\left(V_{\varepsilon}^{s} V_{\varepsilon}^{u} G_{\varepsilon}\right) \circ h_{\varepsilon}^{-1}-V_{\varepsilon}^{s} V_{\varepsilon}^{u} G_{\varepsilon},
$$

but

$$
f_{\varepsilon *} V_{\varepsilon}^{s}=\lambda^{s} V_{\varepsilon}^{s}, \quad f_{\varepsilon *} V_{\varepsilon}^{u}=\lambda^{u} V_{\varepsilon}^{u},
$$

and, since $\lambda^{\mathrm{s}} \lambda^{u}=1$, we have that

$$
\left(V_{\varepsilon}^{s} V_{\varepsilon}^{u} G_{\varepsilon}\right) \circ h_{\varepsilon}^{-1}=V_{\varepsilon}^{s} V_{\varepsilon}^{u}\left(G_{\varepsilon} \circ h_{\varepsilon}^{-1}\right) .
$$

Substituting this in (8) we are led to

$$
V_{\varepsilon}^{s} V_{\varepsilon}^{u}\left(H_{\varepsilon}+G_{\varepsilon}-G_{\varepsilon} \circ h_{\varepsilon}^{-1}\right)=0 .
$$

Since the integral curves of $V_{\varepsilon}^{s}$ are the stable manifolds of $h_{\varepsilon}$, which are dense in $T^{2}$ by Anosov's structural stability theorem, from (9) we get that

$$
V_{\varepsilon}^{u}\left(H_{\varepsilon}+G_{\varepsilon}-G_{\varepsilon} \circ h_{\varepsilon}^{-1}\right)=C_{\varepsilon}^{1}
$$

for some constant $C_{\varepsilon}^{1}$. Evaluating at a maximum of the function between parenthesis, we see that $C_{\varepsilon}^{1}=0$, so

$$
H_{\varepsilon}+G_{\varepsilon}-G_{\varepsilon} \circ h_{\varepsilon}^{-1}=C_{\varepsilon}
$$

for a new constant $C_{\varepsilon}$. Since we have assumed that $\int_{T^{2}} H_{\varepsilon} d x \wedge d y=0$, we have $C_{\varepsilon}=0$. From this we get that for any $x_{\varepsilon} \in T^{2}$ with $h_{\varepsilon}^{N} x_{\varepsilon}{ }^{2}=x_{\varepsilon}$,

$$
\sum_{i=0}^{N-1} H_{\varepsilon}\left(h_{\varepsilon}^{i}\left(x_{\varepsilon}\right)\right)=0
$$

This proves (1), and the proof of Lemma 3 is finished.

Remark. Notice that we do not need $f_{0}$ to be $C^{0}$-conjugate to a linear automorphism, but it suffices if the Lyapunov exponents of periodic points of $f_{0}$ are independent of the point. Since Lemma 2 also holds in general, the part of the theorem dealing with LHI's or GHI's remains true under these apparently weaker assumptions.

Proof of Lemma 4. We take $\varepsilon_{0}>0$ sufficiently small so that each $h_{\varepsilon},-\varepsilon_{0}<\varepsilon<\varepsilon_{0}$, is an Anosov diffeomorphism with stable and unstable vector subbundles $E_{\varepsilon}^{s}, E_{\varepsilon}^{u}$. We shall need the fact that $E_{\varepsilon}^{s}$ and $E_{\varepsilon}^{u}$ are $C^{1+\delta}$ functions defined on $\left[-\varepsilon_{0}, \varepsilon_{0}\right] \times \mathbb{T}^{2}$. This is a consequence of the $C^{r}$-section Theorem 3.2 of [HPS], taking into account the remark on p. 36, as well as Remarks 1 and 2 on p. 38. The mechanism needed to obtain the $C^{1+\delta}$ regularity of $E_{\varepsilon}^{s}$ and $E_{\varepsilon}^{u}$ from this theorem is very standard and we only sketch it. We consider the manifold with boundary $X=\left[-\varepsilon_{0}, \varepsilon_{0}\right] \times \mathbb{T}^{2}$, two 
vector subbundles $\widetilde{E}_{\varepsilon}^{s}, \widetilde{E}_{\varepsilon}^{u}$ of $T \mathbb{T}^{2}$ that are $C^{\infty}$ as functions of $(\varepsilon, x) \in X$ and close to $E_{\varepsilon}^{s}, E_{\varepsilon}^{u}$, and the vector bundle $L\left(\widetilde{E}^{u}, \widetilde{E}^{s}\right)$ over $X$ with fiber over $(\varepsilon, x)$ equal to $L\left(\widetilde{E}_{\varepsilon, x}^{u}, \widetilde{E}_{\varepsilon, x}^{s}\right)$. Now we consider the maps $h: X \rightarrow X, h(\varepsilon, x)=\left(\varepsilon, h_{\varepsilon}(x)\right)$ and $F: L\left(\widetilde{E}^{u}, \widetilde{E}^{s}\right)_{1} \rightarrow L\left(\widetilde{E}^{u}, \widetilde{E}^{s}\right)$ induced by the graph transform of $T h_{\varepsilon}$, that is, $T h_{\varepsilon}(\operatorname{graph}(L))=\operatorname{graph}(F(L))$. Here $L\left(\widetilde{E}^{u}, \widetilde{E}^{s}\right)_{1}$ denotes the bundle of unit balls of $L\left(\widetilde{E}^{u}, \widetilde{E}^{s}\right)$ in some convenient metric that will be specified later. The situation is expressed in the diagram

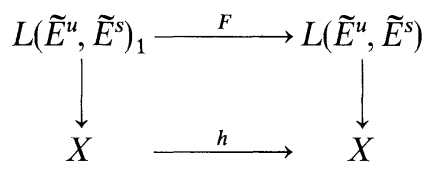

We endow $\mathbb{T}^{2}$ with a metric $\langle\cdot, \cdot\rangle_{\varepsilon}$ adapted to $h_{\varepsilon}$ and we fix on $X$ the metric

$$
\langle\cdot, \cdot\rangle_{(\varepsilon, x)}=\mu \pi_{1}^{*} d \varepsilon^{2}+\pi_{2}^{*}\langle\cdot, \cdot\rangle_{\varepsilon},
$$

where $\pi_{1}: X \rightarrow\left[-\varepsilon_{0}, \varepsilon_{0}\right], \pi_{2}: X \rightarrow \mathbb{T}^{2}$ are the projections and $\mu>0$ is some small number. Now it is easy to check that, if $\widetilde{E}_{\varepsilon}^{u}, \widetilde{E}_{\varepsilon}^{s}$ are sufficiently close to $E_{\varepsilon}^{u}, E_{\varepsilon}^{s}$ and $\mu>0$ sufficiently small, $F$ takes values on $L\left(\widetilde{E}^{u}, \widetilde{E}^{s}\right)_{1}$ and it is a fiber contraction with Lipschitz constant $k_{(\varepsilon, x)}$ in the fiber over $(\varepsilon, x)$ uniformly close to

$$
\left|T_{x} h_{\varepsilon}\right| E^{s}|\cdot|\left(T_{x} h_{\varepsilon}\right)^{-1}\left|E^{u}\right|,
$$

and that $\alpha_{(\varepsilon, x)}=\left|\left(T_{(\varepsilon, x)} h\right)^{-1}\right|$ is unformely close to

$$
\operatorname{Max}\left(\left|\left(T_{x} h_{\varepsilon}\right)^{-1}\right| E^{s}|,|\left(T_{x} h_{\varepsilon}\right)^{-1}\left|E^{u}\right|, 1\right)=\left|\left(T_{x} h_{\varepsilon}\right)^{-1}\right| E^{s} \mid .
$$

If we take $\delta>0$ small, $k_{(\varepsilon, x)} \alpha_{(\varepsilon, x)}^{1+\delta}$ is close to

$$
\left.\left|T_{x} h_{\varepsilon}\right| E^{s}|\cdot|\left(T_{x} h_{\varepsilon}\right)^{-1}\left|E^{u}\right| \cdot\left|\left(T_{x} h_{\varepsilon}\right)^{-1}\right| E^{s}\right|^{1+\delta} .
$$

Since $E_{\varepsilon}^{s}$ has dimension one, this is equal to

$$
\left|\left(T_{x} h_{\varepsilon}\right)^{-1}\right| E^{u}|\cdot|\left(T_{x} h_{\varepsilon}\right)^{-1}\left|E^{s}\right|^{\delta},
$$

and, since $\left|\left(T_{x} h_{\varepsilon}\right)^{-1}\right| E^{u} \mid<\lambda<1$, for some $\delta>0$ we obtain

$$
\sup _{(\varepsilon, x) \in X} k_{(\varepsilon, x)} \alpha_{(\varepsilon, x)}^{1+\delta}<1 .
$$

The theorem referred to in [HPS] allows us to conclude that $F$ has a $C^{1+\delta}$ invariant section. Obviously this section is obtained from $E_{\varepsilon}^{u}$ in the natural way and we can conclude that $E_{\varepsilon}^{u}$ is a $C^{1+\delta}$ function defined on $X$.

Now take two 1-forms $\tilde{\alpha}_{\varepsilon}^{s}, \tilde{\alpha}_{\varepsilon}^{u}$ on $\mathbb{T}^{2}$ such that $E_{\varepsilon}^{s, u}=\operatorname{ker}\left(\tilde{\alpha}^{s, u}\right)$. We can assume they are of class $C^{1+\delta}$ as functions defined on $X=\left[-\varepsilon_{0}, \varepsilon_{0}\right] \times \mathbb{T}^{2}$. We have

$$
h_{\varepsilon}^{*} \tilde{\alpha}_{\varepsilon}^{s, u}=\tilde{\lambda}_{\varepsilon}^{s, u} \tilde{\alpha}_{\varepsilon}^{s, u},
$$

where $\tilde{\lambda}_{\varepsilon}^{s, u}$ are some $C^{1+\delta}$ real functions of $(\varepsilon, x) \in X$ and $\tilde{\lambda}_{0}^{s, u}=\lambda^{s, u}$. We adjust $\tilde{\alpha}_{0}^{s, u}$ so that $\tilde{\alpha}_{0}^{s} \wedge \tilde{\alpha}_{0}^{u}=d x \wedge d y$. The hypothesis of constancy of the Lyapunov exponents implies that for an $N$-periodic point $x_{\varepsilon}$ of $h_{\varepsilon}$ we have

$$
\frac{1}{N} \sum_{j=1}^{N} \log \tilde{\lambda_{\varepsilon}^{s, u}}\left(h_{\varepsilon}^{j}\left(x_{\varepsilon}\right)\right)=\log \lambda^{s, u},
$$


and by Livsic theorem [L], we can take a Hölder solution $\Phi_{\varepsilon}^{s, u}$ of

$$
\Phi_{\varepsilon}^{s, u}\left(h_{\varepsilon}\right)-\Phi_{\varepsilon}^{s, u}=\log \left(\lambda^{s, u} / \widetilde{\lambda}_{\varepsilon}^{s, u}\right) .
$$

The proofs of Theorems 2.1 and 2.2 of [LMM] show that $\Phi_{\varepsilon}^{s, u_{i}} C^{1+\delta}$ as a function defined on $X$. We also normalize $\Phi_{0}^{s, u}=0$. Now we define the 1 -forms

$$
\alpha_{\varepsilon}^{s, u}=e^{\Phi_{\varepsilon}^{s, u}} \tilde{\alpha}_{\varepsilon}^{s, u}
$$

of class $C^{1+\delta}$ on $X$ such that

$$
h_{\varepsilon}^{*} \alpha_{\varepsilon}^{s, u}=\lambda^{s, u} \alpha_{\varepsilon}^{s, u} .
$$

We shall prove that $\alpha_{\varepsilon}^{s, u}$ are closed forms. Let $V_{\varepsilon}^{s, u}$ be the dual basis of $\alpha_{\varepsilon}^{s, u}$. From the last equality we have

$$
h_{\varepsilon *} V_{\varepsilon}^{s, u}=\lambda^{s, u} V_{\varepsilon}^{s . u}
$$

and, since $\lambda^{s} \lambda^{u}=1$

$$
h_{\varepsilon *}\left[V_{\varepsilon}^{s}, V_{\varepsilon}^{u}\right]=\left[V_{\varepsilon}^{s}, V_{\varepsilon}^{u}\right] .
$$

By hyperbolicity this implies $\left[V_{\varepsilon}^{s}, V_{\varepsilon}^{u}\right]=0$, and this immediately leads to $d x_{\varepsilon}^{s, u}=0$.

Since $h_{\varepsilon \#}=h_{0 \#}: H^{1}\left(\mathbb{T}^{2}, \mathbb{R}\right) \rightarrow H^{1}\left(\mathbb{T}^{2}, \mathbb{R}\right)$ is constant and hyperbolic, we can introduce some multiplicative factor in the definition of $\alpha_{\varepsilon}^{s, u}$ so that the classes $\left[\alpha_{\varepsilon}^{s . u}\right]$ are constant. To obtain (b) it suffices to observe that $\alpha_{\varepsilon}^{s} \wedge \alpha_{\varepsilon}^{u}$ is an invariant volume element for $h_{\varepsilon}$, and then it is a constant times $d x \wedge d y$. Since the class $\left[\alpha_{\varepsilon}^{s} \wedge \alpha_{\varepsilon}^{u}\right] \in H^{2}\left(\mathbb{T}^{2}, \mathbb{R}\right)$ is constant, $\alpha_{\varepsilon}^{s} \wedge \alpha_{\varepsilon}^{u}$ is cohomologous to $d x \wedge d y$, and we obtain (b). This finishes the proof of Lemma 4.

Remark. The previous theorem is still true in the context of real analytic isotopies of real analytic diffeomorphisms. The proof is the same, except for the application of the lemmata, where one uses Theorem 1.4 from [LMM] instead of Theorem 1.3.

Remark. Notice that since each $h_{\varepsilon}$ preserves area, $\delta$ can be taken as close to 1 as we want in Lemma 4.

Acknowledgements. We thank A. Katok and J. Palis, from whom we knew about the $C^{1+o}$ regularity of codimension one foliations. Particularly, J. Palis told us how this was a consequence of the classical work by Hirsch, Pugh, and Shub.

We also wish to thank R. de la Llave for useful conversations and encouragement.

\section{References}

[A] Anosov, D.A.: Geodesic flows on riemannian manifolds with negative curvature. Proc. Steklov Inst. 90, 1-235 (1967)

[Ar] Arnold, V.I.: Geometrical methods in the theory of ordinary differential equations. Berlin, Heidelberg, New York: Springer 1983

[BK] Burns, K., Katok, A.: Manifolds with non-positive curvature. Preprint, Mathematical Sciences Research Institute, Berkeley 1984

[CEG] Collet, P., Epstein, H., Gallavotti, G.: Perturbations of geodesic flows on surfaces of constant negative curvature and their mixing properties. Commun. Math. Phys. 95, $61-112(1984)$ 
[GK] Guillemin, V., Kazdan, J.: Some inverse spectral results for negatively curved 2-manifolds. Topology 19, 301-313 (1980)

[HPS] Hirsch, M.W., Pugh, C.C., Shub, M.: Invariant manifolds. Lecture Notes in Mathematics, Vol. 583. Berlin, Heidelberg, New York: Springer 1977

[L] Livsic, A.: Cohomology properties of $Y$ systems. Math. Notes 10, 754757 (1971)

[LMM] Llave, R. de la, Marco, J.M., Moriyón, R.: Canonical perturbation theory of Anosov systems and regularity results for Livsic cohomology equation. Ann. Math. 123, 537-611 (1986)

[M] Manning, A.: There are no new Anosov diffeomorphisms on tori. Am. J. Math. 96, 422-429 (1974)

[Mo] Moser, J.: On the volume elements on a manifold. Trans. Am. Math. Soc. 120, 286-294 (1965)

[P] Pesin, Ya.B.: Lyapunov characteristic exponents and smooth ergodic theory. Russ. Math. Surv. 32, 55-114 (1977)

[S] Smale, S.: Differentiable dynamical systems. Bull. Am. Math. Soc. 73, 747-817 (1967)

Communicated by J. Mather

Received February 17, 1986; in revised form September 22, 1986 
